\title{
Chemical State Mapping of Amorphous Carbon Films by Soft X-ray Emission
}

\section{Spectroscopy}

\author{
Masami Terauchi ${ }^{1 *}$ and Yohei Sato ${ }^{1}$ \\ ${ }^{1 .}$ Institute of Multidisciplinary Research for Advanced Materials, Tohoku University, Sendai, Japan. \\ * Corresponding author: terauchi@m.tohoku.ac.jp
}

Soft X-ray emissions, which originate from electronic transitions from valence bands (bonding electron states) to inner-shell electron levels, inform us energy states of bonding electrons if those energies are analyzed with an energy resolution better than $1 \mathrm{eV}$. Thus, soft-X-ray emission spectroscopy (SXES) based on electron microscopy should be a hopeful method to evaluate bonding states of identified small specimen areas. SXES can apply to metal, semiconductor and insulator materials under a conventional vacuum condition of electron microscopes. One drawback is its low detection efficiency. Then, a commercial SXES spectrometer, which was composed of varied line spacing (aberration corrected) gratings and an area detector, was designed as an attachment for EPMA/SEM [1], which can use a larger probe current than that of TEM. As EPMA/SEM are operated at a lower accelerating voltage than that of TEM, bonding states of irradiation sensitive materials can be investigated. Here, SXES imaging of chemical state mapping of carbon atoms of amorphous carbon nitride (a-CNx) films and diamond like carbon (DLC) films are presented [2,5].

a-CNx films show a controllability of photoconductivity and UV luminescence by a change of the content of nitrogen $x[3,4]$. First, high energy-resolution EELS was applied at $80 \mathrm{kV}$. It was notice a change of valence electron excitation spectra due to irradiation damage. Then, EPMA-SXES was applied at $5 \mathrm{kV}$. At the voltage, any change of carbon K-emission spectrum intensity was not detected. Figure 1(a) shows a carbon K-emission spectrum of a-CNx ( $2^{\text {nd }}$ order), which shows two peak structure [2]. $3^{\text {rd }}$ order $\mathrm{N} \mathrm{K}$-emission intensity is also seen. Peak energies of A and B are the same with those of graphite ( $\mathrm{sp}^{2}-\sigma$ bonding) and diamond ( $\mathrm{sp}^{3}-\sigma$ bonding). a-CNx film with a smaller $\mathrm{x}$ showed a smaller B intensity. Thus, inclusion of $\mathrm{N}$ induces a presence of $\mathrm{sp}^{3}$-bonding. This is also linked with an increase of macroscopic electrical resistivity of those films. Figure 1(b) and (c) show intensity distribution of energy windows of $\mathrm{I}_{\mathrm{A}}$ and $\mathrm{I}_{\mathrm{B}}$, indicated in Fig.1(a). Pixel size is $1.2 \mu \mathrm{m}^{2}$. The intensity variation in left and right figures correspond to variations of $\mathrm{sp}^{2}-\mathrm{C}$ and $\mathrm{sp}^{3}-\mathrm{C}$ bonding signals, respectively. Thus, a brighter pixel area in the right figure includes a larger amount of $\mathrm{sp}^{3}$-bonding. This experimental result shows that the high energy-resolution soft X-ray mapping with an appropriate energy window can show not only elemental distribution but also chemical bonding state distribution of bulk materials [2].

Figure 2(a) shows $2^{\text {nd }}$ order C K-emission spectrum of a DLC film formed on Si wafer. From XPS analysis of $\mathrm{C}-1 \mathrm{~s}$ energy level, this film was assigned to have a ratio of $\mathrm{sp}^{3}-\mathrm{C} / \mathrm{sp}^{2}-\mathrm{C}=2.42$. C K-emission spectra of graphite and diamond are also shown for comparison. As the DLC film composed of $\mathrm{sp}^{3}-\mathrm{C}$ and $\mathrm{sp}^{2}-\mathrm{C}$, the peak energy of C-K emission of this DLC film is placed between those of graphite $\left(\mathrm{sp}^{2}-\right.$ C) and diamond $\left(\mathrm{sp}^{3}-\mathrm{C}\right)$. When setting energy windows of $139-141 \mathrm{eV}$ for $\mathrm{sp}^{3}-\mathrm{C}$ and $130-143 \mathrm{eV}$ for all $\mathrm{C}-\mathrm{K}$ intensity and calculate a ratio of $\mathrm{I}(139-141 \mathrm{eV}) / \mathrm{I}(130-143 \mathrm{eV})$, a distribution image of $\mathrm{sp}^{3}-\mathrm{C}$ is obtained as shown in Fig.2(b). The ratio variates $\pm 6 \%$ within the imaged area. This map shows an amount of $\mathrm{sp}^{3}-\mathrm{C}$ bond variate in a few $\mu \mathrm{m}$ scale [5]. 


\section{References:}

[1] H Takahashi et al., Microscopy and Microanalysis 19 (S2) (2013), p. 1258.

[2] S Ishii et al., Microscopy 67 (2018), p. 244.

[3] S Nitta et al., Journal of Non-Crystalline Solids 227-230 (1998), p. 655.

[4] Y Iwano et al., Japanese Journal of Applied Physics 47 (2008), p. 7842.

[5] Results of a-CNx and DLC are collaborated ones with Prof. Aono of Kagoshima Univ. and Prof.

Takakuwa of Tohoku Univ., respectively. Those works were partly supported by the Research Program of "Dynamic Alliance for Open Innovation Bridging Human, Environment and Materials" in "Network Joint Research Centre for Materials and Devices".
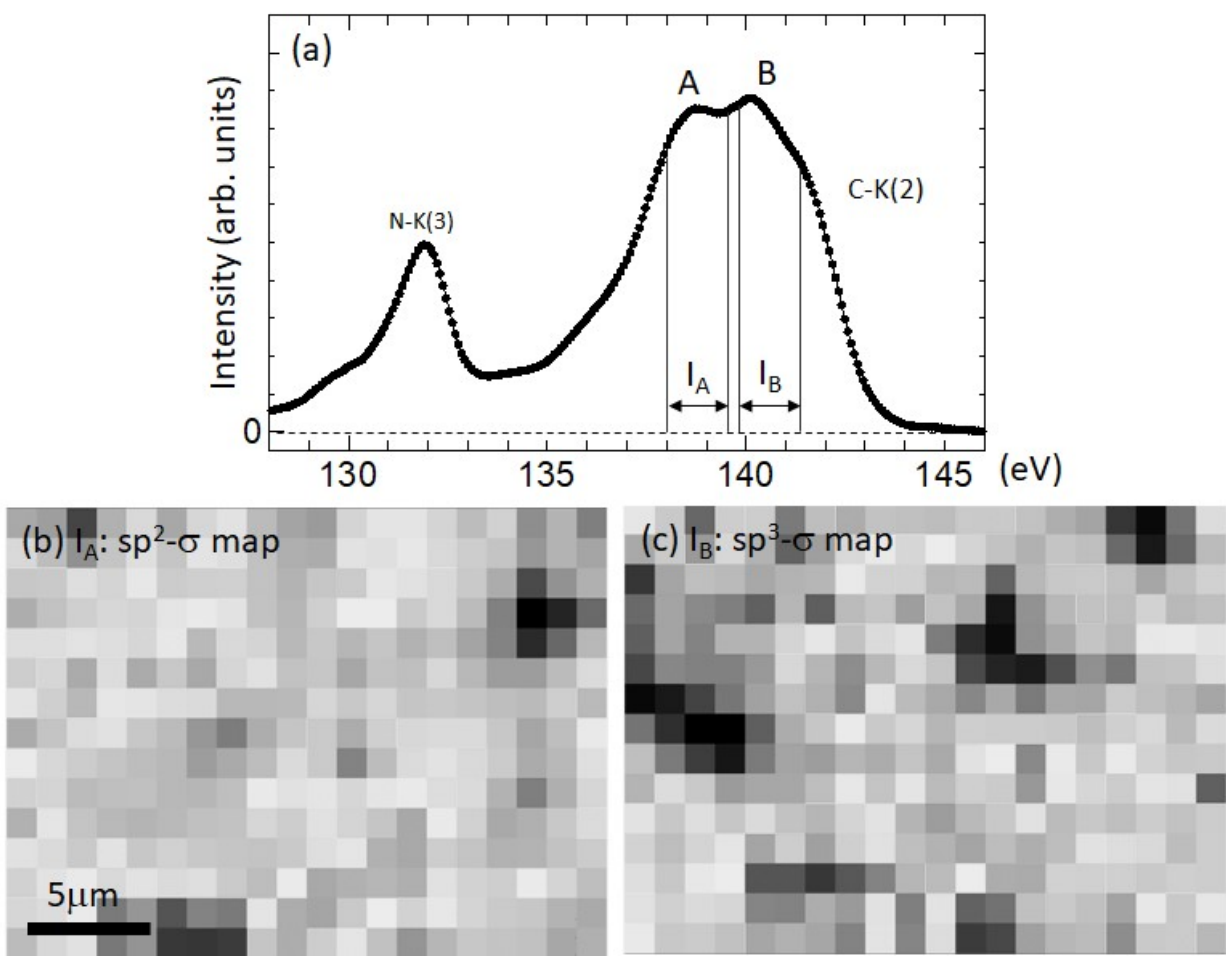

Figure 1. (a) $2^{\text {nd }}$ order $\mathrm{C}$ K-emission spectrum of a-CNx, (b) and (c) respectively show intensity distributions of $\mathrm{I}_{\mathrm{A}}\left(\mathrm{sp}^{2}-\sigma\right.$ bonding) and $\mathrm{I}_{\mathrm{B}}\left(\mathrm{sp}^{3}-\sigma\right.$ bonding) indicated in (a).
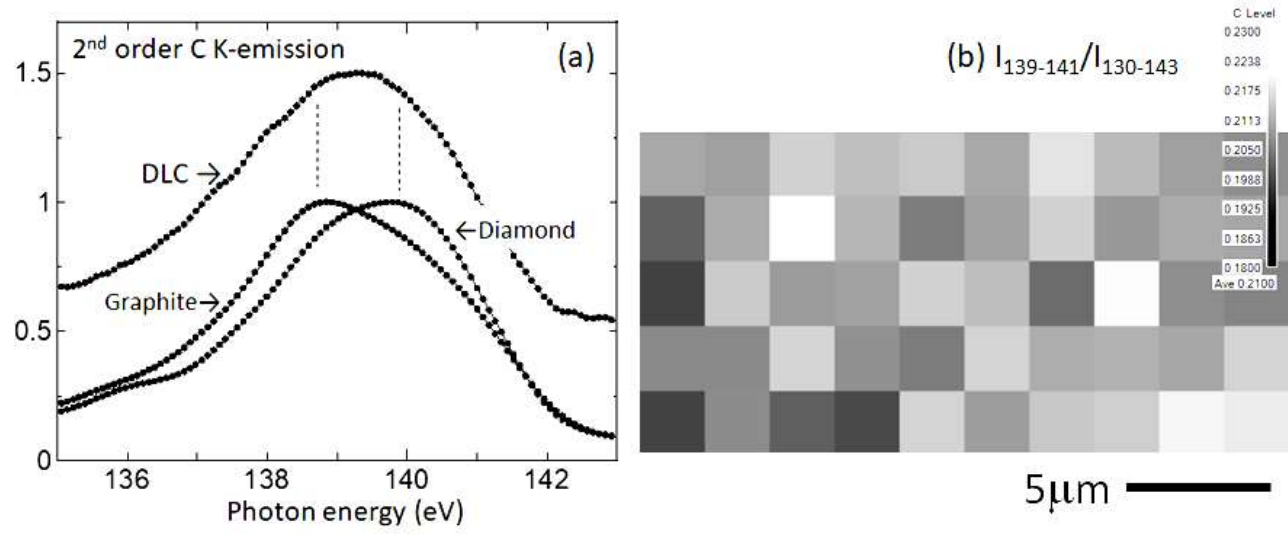

Figure 2. (a) $2^{\text {nd }}$ order C K-emission spectra of a DLC film, graphite and diamond, (b) spacial variation of a ratio $\mathrm{I}(139-141) / \mathrm{I}(130-143)$. 